\title{
Figure $\mathrm{A}$
}

negative regulation of transcription response to endoplasmic reticulum stress

ER-nuclear signaling pathway

cell division

forebrain development regulation of transcription intracellular transport

negative regulation of macromolecule metabolic... transcription

protein localization

ubiquitin-dependent protein catabolic process

organelle localization protein targeting

negative regulation of gene expression

M phase

establishment of protein localization protein transport organelle fission $M$ phase of mitotic cell cycle cell cycle phase mitosis nuclear division mitotic cell cycle cell cycle process cell cycle

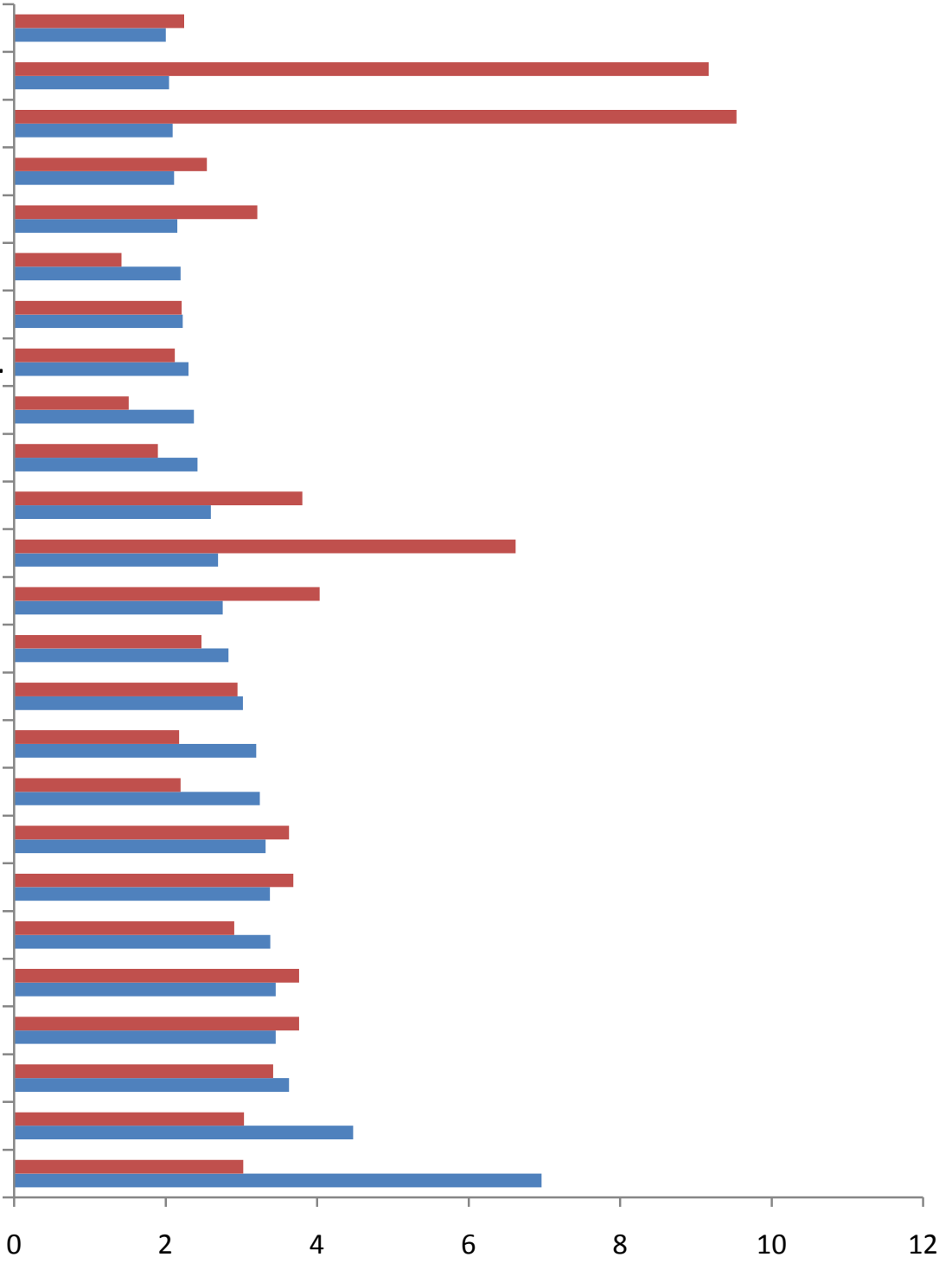

Fold Enrichment -LOG(Pvalue)

Figure A in S2 File. GO analysis of 334 genes with upregulated proximal pA in GSCs. 


\section{Figure B}

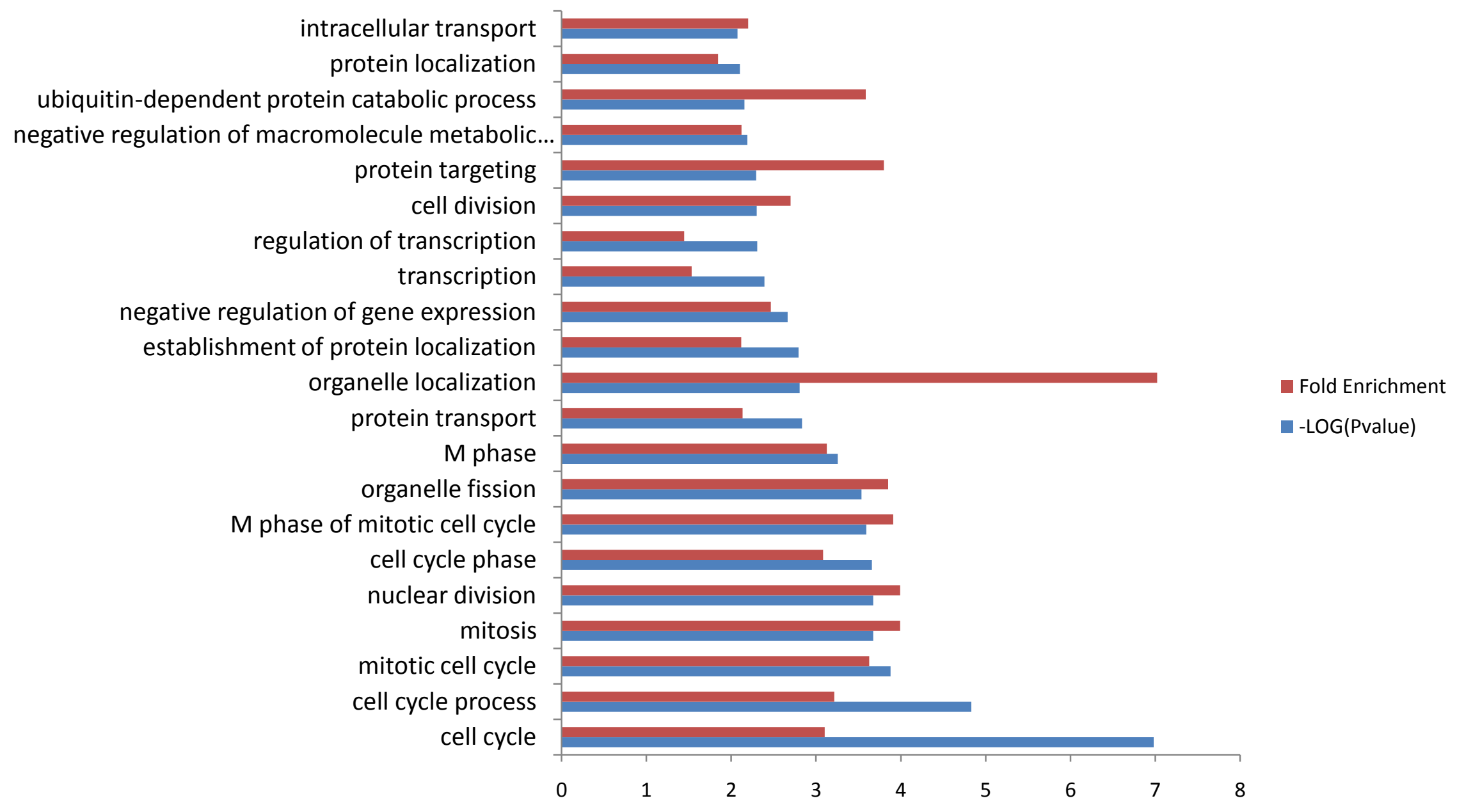

Figure B in S2 File. GO analysis of 239 genes with 3'UTR distal-to-proximal switch events happened both in GSCs vs MEFs and ESCs vs MEFs comparisons. 


\section{Figure $\mathrm{C}$}

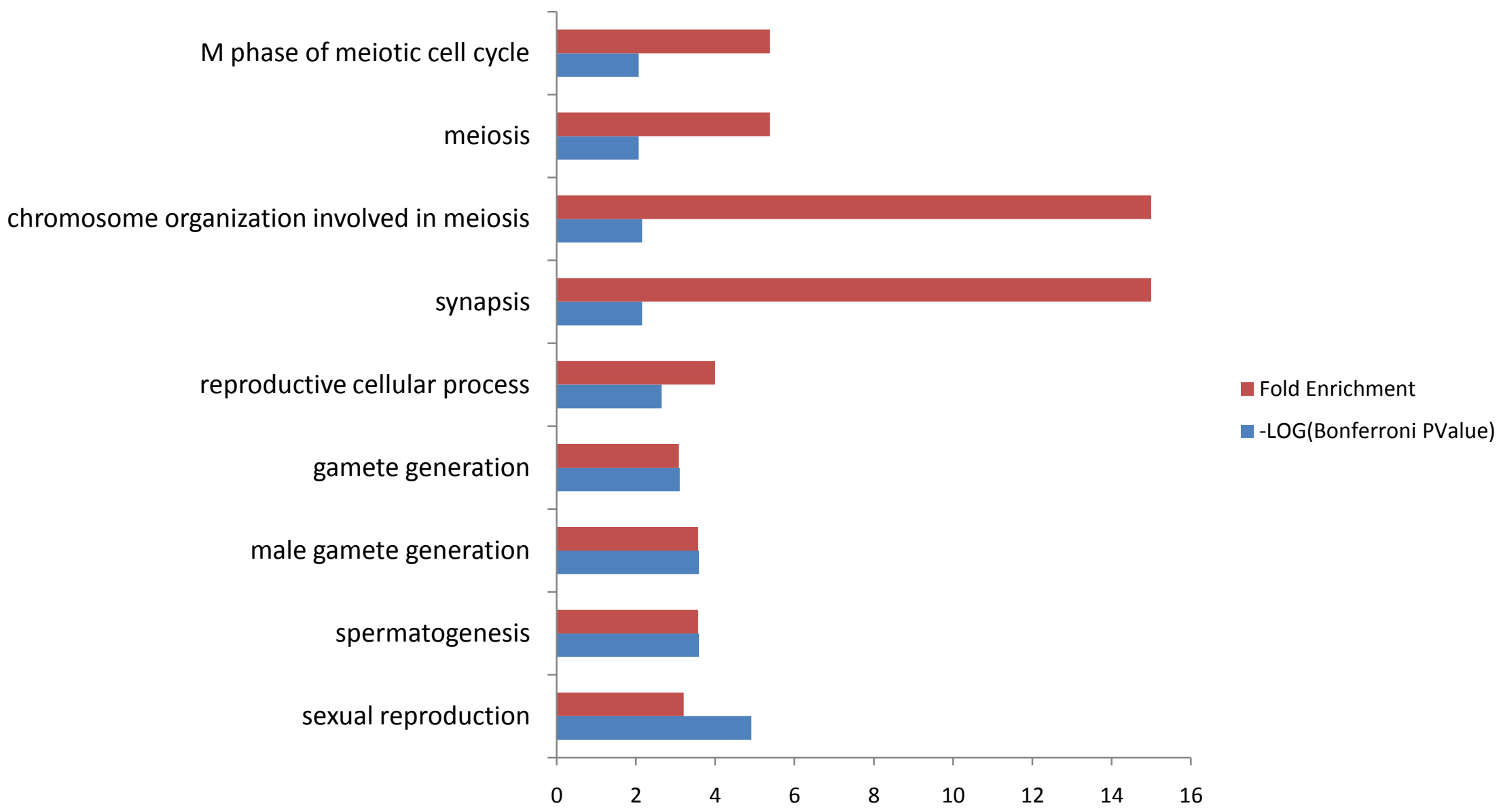

Figure C in S2 File. GO analysis of 643 GSCs and ES up-regulated genes as compare to MEFs. 


\section{Figure D}

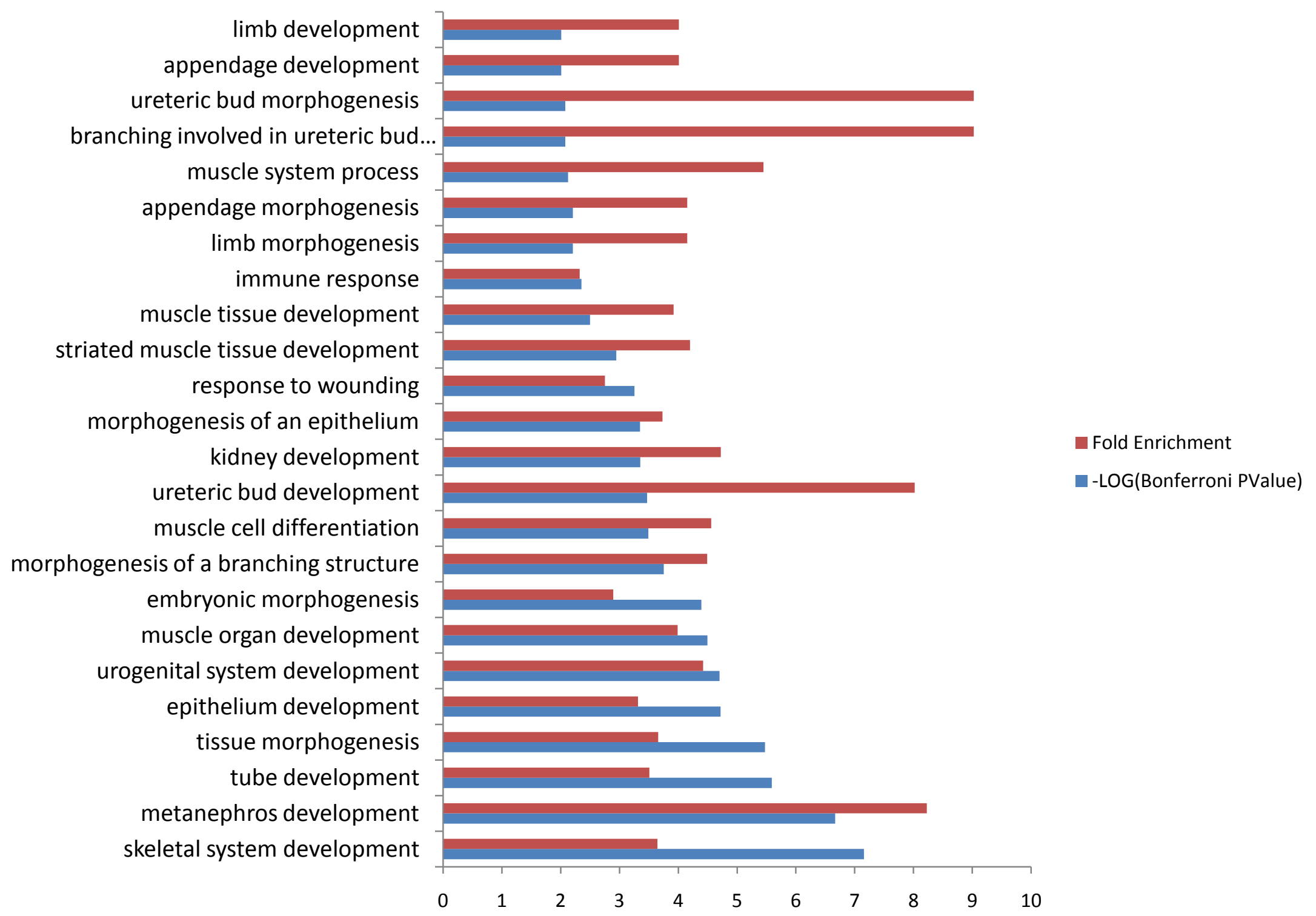

Figure D in S2 File. GO analysis of 718 GSCs and ES down-regulated genes as compare to MEFs. 


\section{Figure $E$}

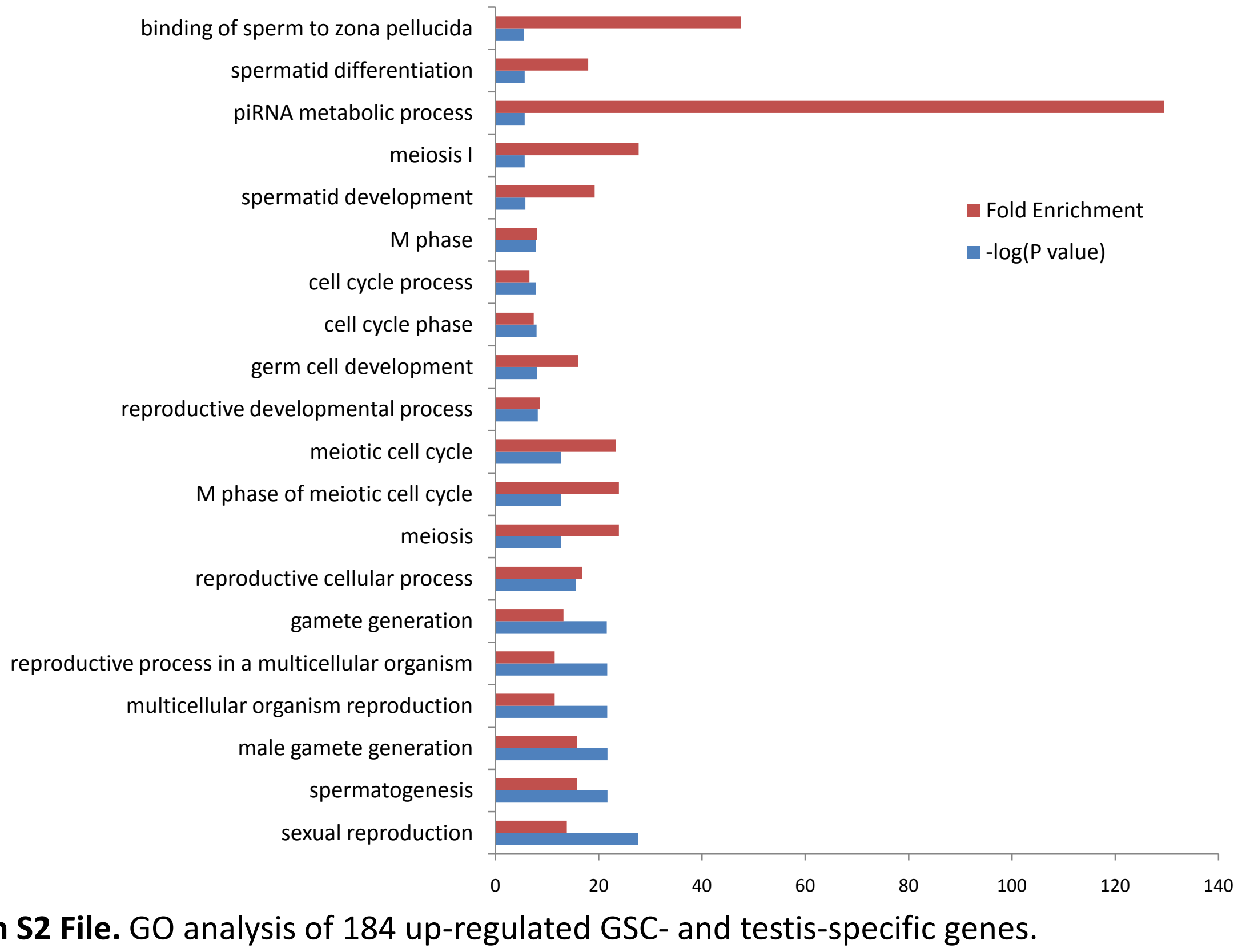

Figure E in S2 File. GO analysis of 184 up-regulated GSC- and testis-specific genes. 


\section{Figure $F$}

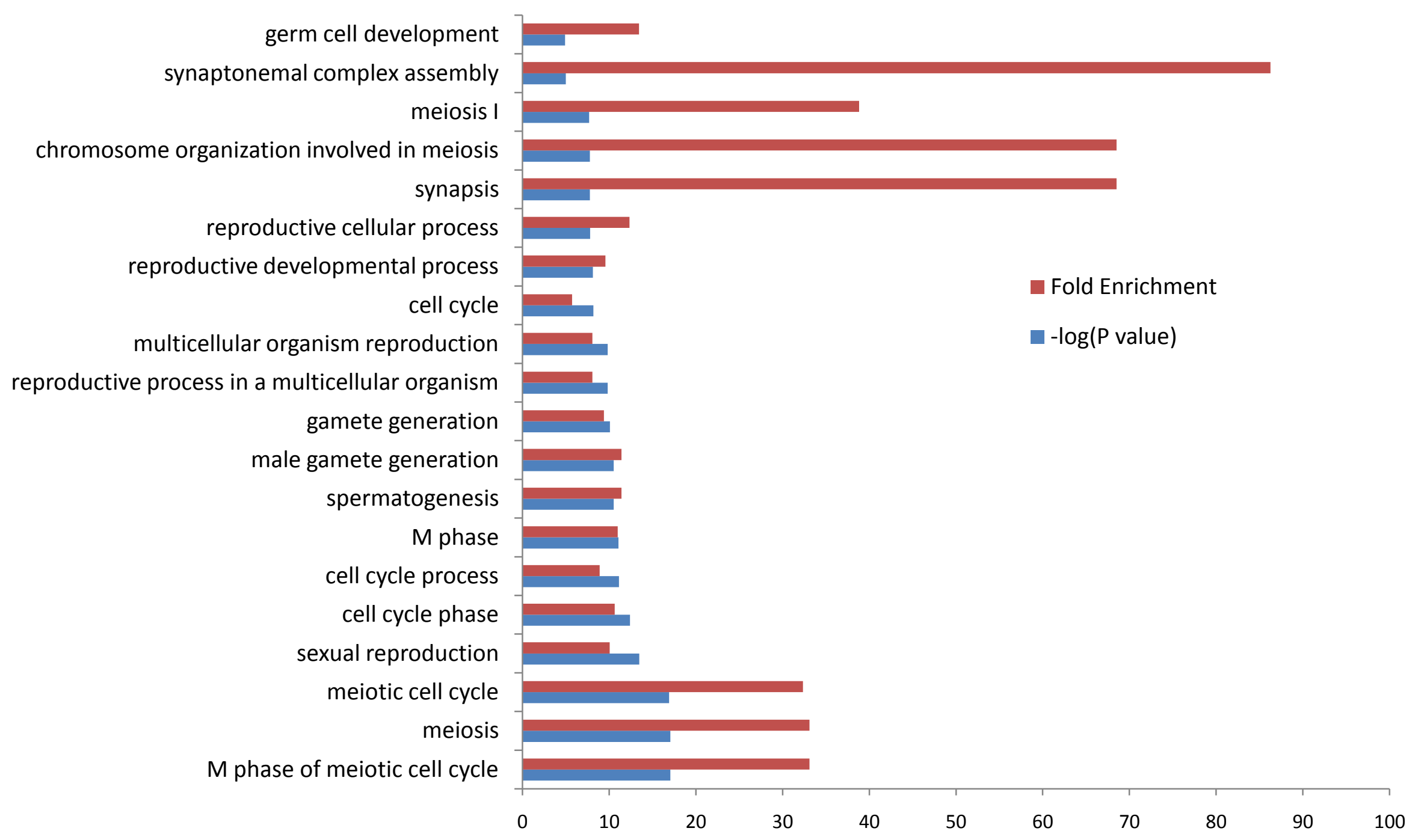

Figure $\mathbf{F}$ in S2 File. GO analysis of 125 up-regulated meiosis-specific genes in GSCs. 


\section{Figure $\mathbf{G}$}

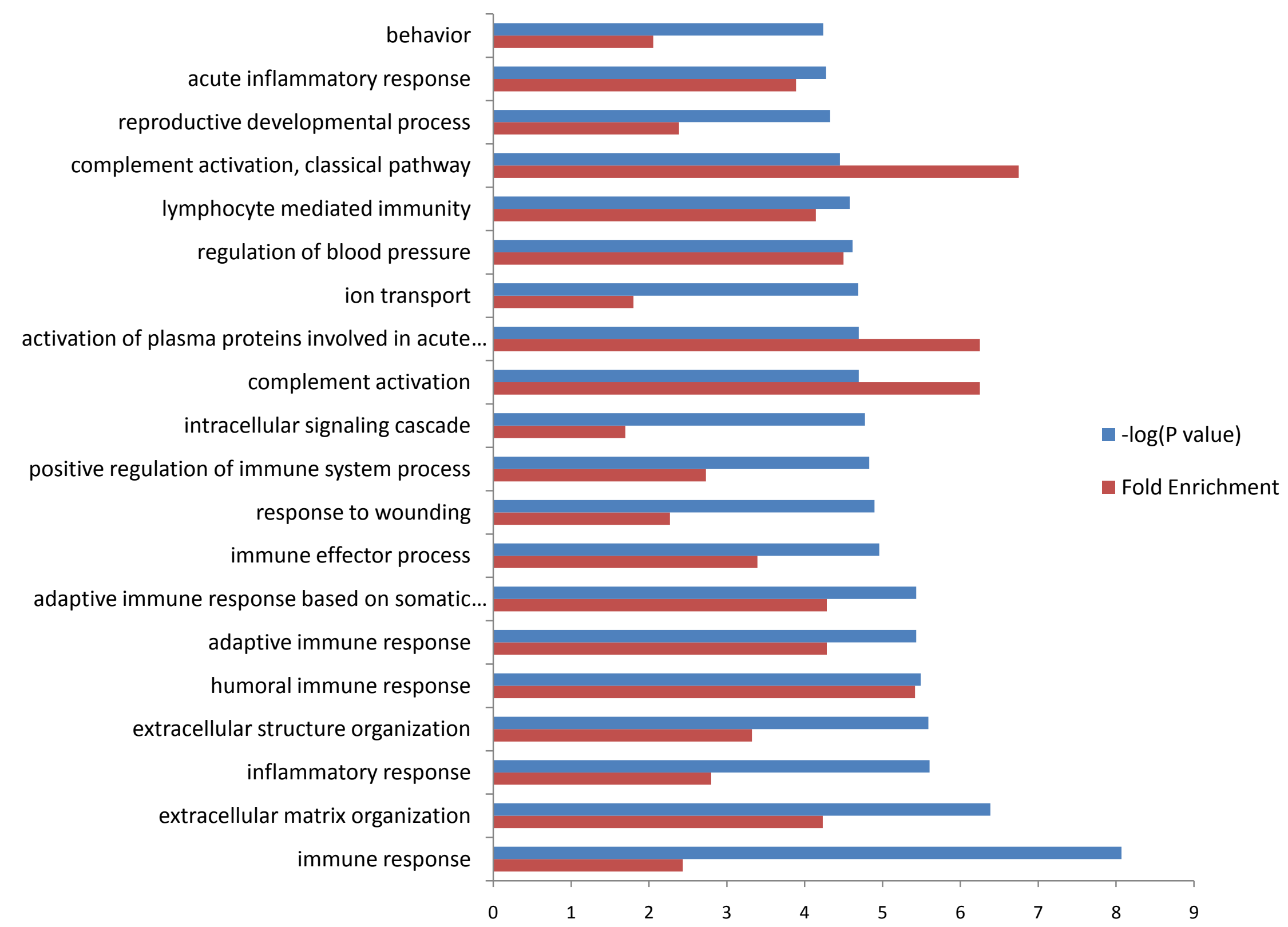

Figure $\mathbf{G}$ in S2 File. GO analysis of 1070 up-regulated non meiosis-specific genes in GSCs. 


\section{Poly(A) site mostly used in GSCs}
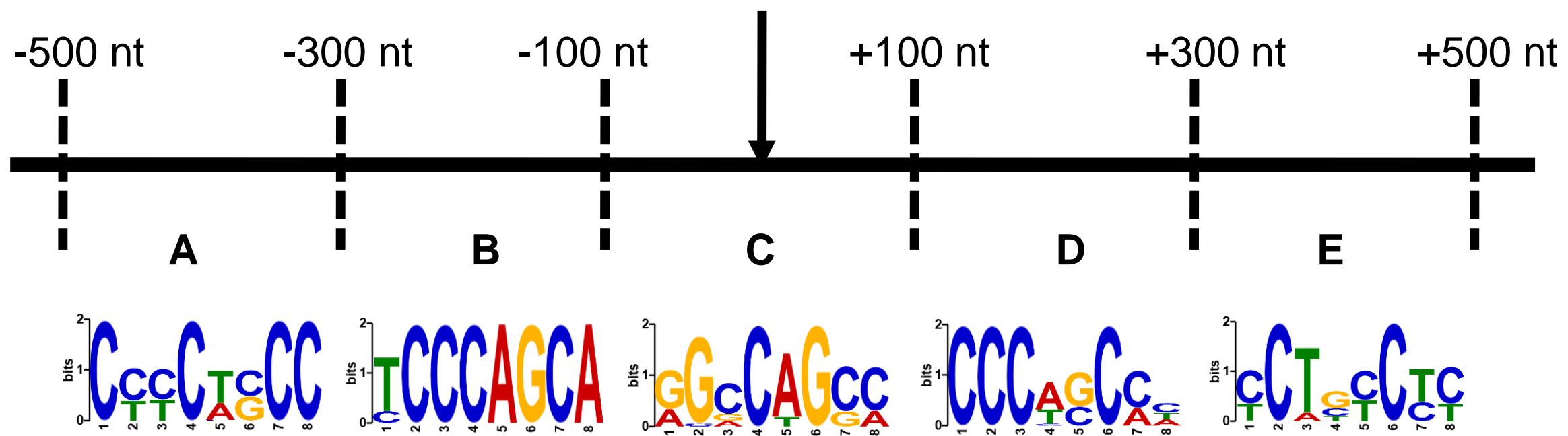

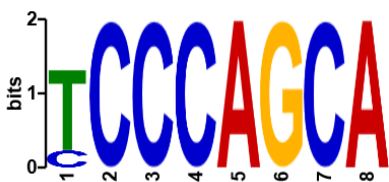

$9.6 \mathrm{e}-015$

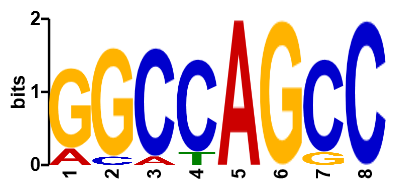

$4.8 \mathrm{e}-009$

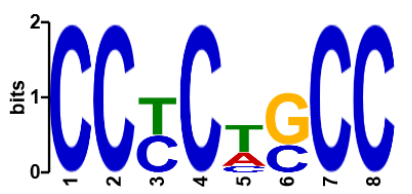

1.3e-006

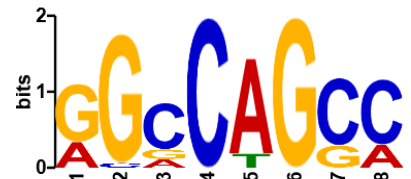

3.3e-009

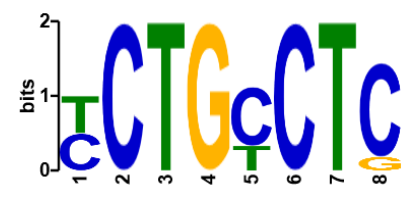

$1.1 \mathrm{e}-008$

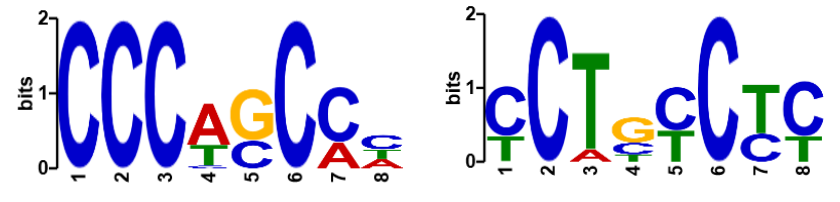

6.0e-017

Figure $\mathbf{H}$ in S2 File. Cis elements analysis for APA. Five regions surrounding the poly $(A)$ site used preferentially in GSCs compared to ESCs and MEFs were shown. Five subregions $A, A, C, D, E$ were devided in the -500 and +500 to the APA site. Motifs were analyzed using Multiple Em for Motif Elicitation (MEME) Software. E values representing the statistical significance of the motif were shown below. The most statistically significant (low E-value) motifs were shown first. 